\title{
Indications and Complications of Flexible Fiberoptic Bronchoscopy in Children: A 5-Year Experience at a Tertiary Care Hospital in Iran
}

\author{
Rohola Shirzadi (iD ${ }^{1,2}$, Safoura Navaei ${ }^{1,2}$, Niloofar Razavi-Khorasani ${ }^{3}$, Farzad Masiha ${ }^{4}$, Seyed Hossein \\ Mirlohi ${ }^{1,2}$, Mahya Mohamadi ${ }^{2}$, Alireza Ebrahimsoltani ${ }^{5}$ and Mohammad Reza Modaresi ${ }^{1,2, *}$ \\ ${ }^{1}$ Pediatric Respiratory and Sleep Medicine Research Center, Children's Medical Center, Tehran University of Medical Sciences,Tehran, Iran \\ ${ }^{2}$ Department of Pediatrics, Tehran University of Medical Sciences,Tehran, Iran \\ ${ }^{3}$ Network of Immunity in Infection, Malignancy and Autoimmunity (NIIMA), Universal Scientific Education and Research Network (USERN), Tehran, Iran \\ ${ }^{4}$ Department of Pediatrics, Mazandaran University of Medical Sciences, Sari, Iran \\ ${ }^{5}$ Department of Anesthesiology, Tehran University of Medical Sciences, Tehran, Iran \\ "Corresponding author: Pediatric Respiratory and Sleep Medicine Research Center, Children's Medical Center, Tehran University of Medical Sciences, Tehran, Iran. Email: \\ moh.modaressi@gmail.com
}

Received 2019 April 23; Revised 2020 January 24; Accepted 2020 March 18.

\begin{abstract}
Background: Flexible fiberoptic bronchoscopy (FFB) is known as an important diagnostic and therapeutic modality in the evaluation of respiratory disorders in pediatric population.

Objectives: The study aims to highlight common indications and risk of complications associated with bronchoscopy in our population.

Methods: This retrospective evaluation was performed in all patients that underwent flexible bronchoscopy at Children's Medical Center (affiliated to Tehran University of Medical Sciences) between April 2011 and September 2016.

Results: Of 800 bronchoscopies, 574 (71.7\%) were performed for diagnostic and 226 (28.2\%) for therapeutic purposes. Major indications included radiographic abnormalities (30\%), a foreign body or suspected foreign body (28.5\% of all FFBs) and stridor or wheezing $(25 \%$ of all $\mathrm{FBB})$. The incidence of major complications associated with FFBs was $0.87 \%$. The most frequent complication was pneumothorax, followed by lung hemorrhage, and respiratory failure.

Conclusions: Our findings support early intervention and utilization of bronchoscopy in the pediatric population with variable respiratory complaints.
\end{abstract}

Keywords: Flexible Fiberoptic Bronchoscopy, Pediatric Pulmonary Disease, Respiratory Tract, Children

\section{Background}

Flexible fiberoptic bronchoscopy (FFB) is recognized as an important diagnostic and therapeutic modality in the evaluation of respiratory disorders in pediatric population $(1,2)$. Since its first introduction in late 70 , the feasibility and safety of this technique has been improved and continues to evolve which allowed physicians to use this procedure frequently for different indications (3). FFB has been widely used for identifying the cause of bronchiectasis, recurrent wheezing and persistent stridor $(1,2,4)$. Immunocompromised patients and those with severe asthma are two main groups at risk for respiratory complications, for which a quick and accurate diagnosis is crucial (5-7). Although, bronchoscopy is considered a safe and effective diagnostic tool, it is associated with a number of mechanical complications arising from airway trauma. Other potential complications are due to anesthesia, desaturation, laryngeal spasm and patient comorbidities (8-10). Limited data exists on bronchoscopy-related complications among pediatric patients from developing countries. Therefore, the objectives of the present study were to evaluate the indications for FFB, patients' characteristics and rate of complications.

\section{Objectives}

The study aims to highlight common indications and risk of complications associated with bronchoscopy in our population.

\section{Methods}

\subsection{Patients}

The present study is a retrospective analysis of a total number of 842 patients who underwent flexible bron- 
choscopy at Children's Medical Center (a tertiary pediatric hospital affiliated to Tehran University of Medical Sciences). This study took place between April 2011 and September 2016. Data on all bronchoscopic procedures were collected and analyzed. Data regarding patients' characteristics, indications for bronchoscopy, radiographic findings, endoscopic findings, bronchoalveolar lavage (BAL) analysis and all complications were extracted by reviewing the patient's charts and bronchoscopy reports. The inclusion criteria for patients undergoing FFB were as follows: persistent wheezing, stridor preceding the acute presentation, and upper and lower airway disease and pulmonary disease. Patients with any episode of, hypotension, arrhythmias and unstable hemodynamic were excluded from this study. Ethics approval from research ethics committee at Tehran University of Medical Sciences was obtained. All parents were asked to fill an informed consent before all procedures were performed. The process of this study was conducted in accordance with the Declaration of Helsinki.

\subsection{Bronchoscopy Procedure}

The method and description of our FFB procedure have been explained in previous studies (11). In summary, FOB (EVIS EXERA III CLV-190) with a diameter of 2.8 and $3.5 \mathrm{~mm}$ was performed under instilling local or general anesthesia without the use of muscle relaxants. Endotracheal intubation was performed for all patients by an experienced anesthesiologist. In the case of difficult intubating conditions, FFB was inserted to identify the glottic opening. Then, endotracheal tube was placed at the beginning of the FFB and threaded both together until they reached the optimum position of $1.5 \mathrm{~cm}$ above the carina. After confirming the correct positioning of the tube, FFB was performed. Intravenous lines were established for all patients and continuous cardiorespiratory monitoring was instituted. All subjects were kept under close observation following the completion of the procedure for monitoring potential complications. Anesthesia was provided by an experienced pediatric anesthetist. Fasting prior to the procedure lasted at least 6 hours before anesthesia. Sevoflurane was used as an anesthetic drug. Intravenous atropine $(0.01 \mathrm{mg} / \mathrm{kg})$ was given to patients before the procedure. Topical anesthesia of the vocal cords and trachea were provided with $1 \%$ lidocaine. Following the procedure, intravenous fluids were administered and patient was maintained NPO for at least 6 hours with monitoring of vital signs every 15 minutes after patient regained consciousness. BAL was performed for various indications according to ERS guidelines (12). BAL was accomplished by administration of sterile saline (1-3 mL/kg) and wedging the scope, followed by immediate aspiration via the suction channel of the broncho- scope into a sterile container. Airway foreign bodies were extracted using a retrieval basket. In rare cases of large foreign bodies, rigid bronchoscopy was used. In cases with persistent atelectasis caused by full mucus plug blockages, $\mathrm{N}$-acetylcysteine and hypertonic saline were used via the bronchoscopic port for clearance of lung mucosal secretions and suctioned directly through the working channel.

\subsection{Statistical Analysis}

The data was analyzed using proportions. The Pearson correlation was used to examine the association between variables and occurrence of FFB-related complications. P values less than 0.05 were considered as statically significant. Data were analyzed using SPSS Statistics V.17.0 (Chicago, Illinois).

\section{Results}

A total number of 842 pediatric patients including 581 males and 259 females underwent FFB over a 5 year period. Among the 842 procedures performed, 800 medical records were gathered and evaluated, while the data of 42 records were either missing or incomplete. Of the 800 bronchoscopies performed, 574 (71.7\%) were performed for diagnostic purposes and 226 (28.2\%) for therapeutic purposes. The age distribution of subjects is demonstrated in Figure 1. The mean age was 3.5 years (range: 0.01 to 17 years). Difficult intubation was found in 39 (4.6\%) cases and no complication was observed following FFB-assisted intubation among these patients.

Indications for diagnostic bronchoscopy are summarized in Table 1. Major indications included radiographic abnormalities (30\%), a foreign body or suspected foreign body (28.5\% of all FFBs), stridor or wheezing (25\% of all FBB), suspected lower airway infections (15.5\%), persistent atelectasis or emphysema (10.3\%), bronchoalveolar lavage (5.5\%) and the diagnostic evaluation of chronic cough (4.3\%). The overall diagnostic yield was 68.3\% (547/800). Of 228 patients suspected to have foreign body aspiration, 156 (68.8\%) patients were confirmed. For therapeutic purposes, the major indications included suctioning or extraction of foreign bodies (28.5\%), retained secretions and mucus plugs (1.37\% of all FBBs) and assisting with difficult intubations ( $0.5 \%$ ) (Table 1$)$. A total of 36 patients (4.5\%) developed complications during FFB procedures. No mortality has been experienced in our center. The incidence of major complications associated with FFBs was $0.87 \%$. The most frequent complication was pneumothorax, followed by lung hemorrhage, and respiratory failure (Table 2). In patients with evidence of persistent low oxygen levels, high peak inspiratory pressure (PIP) was used to maintain oxygen saturation that resulted in pneumothorax in 


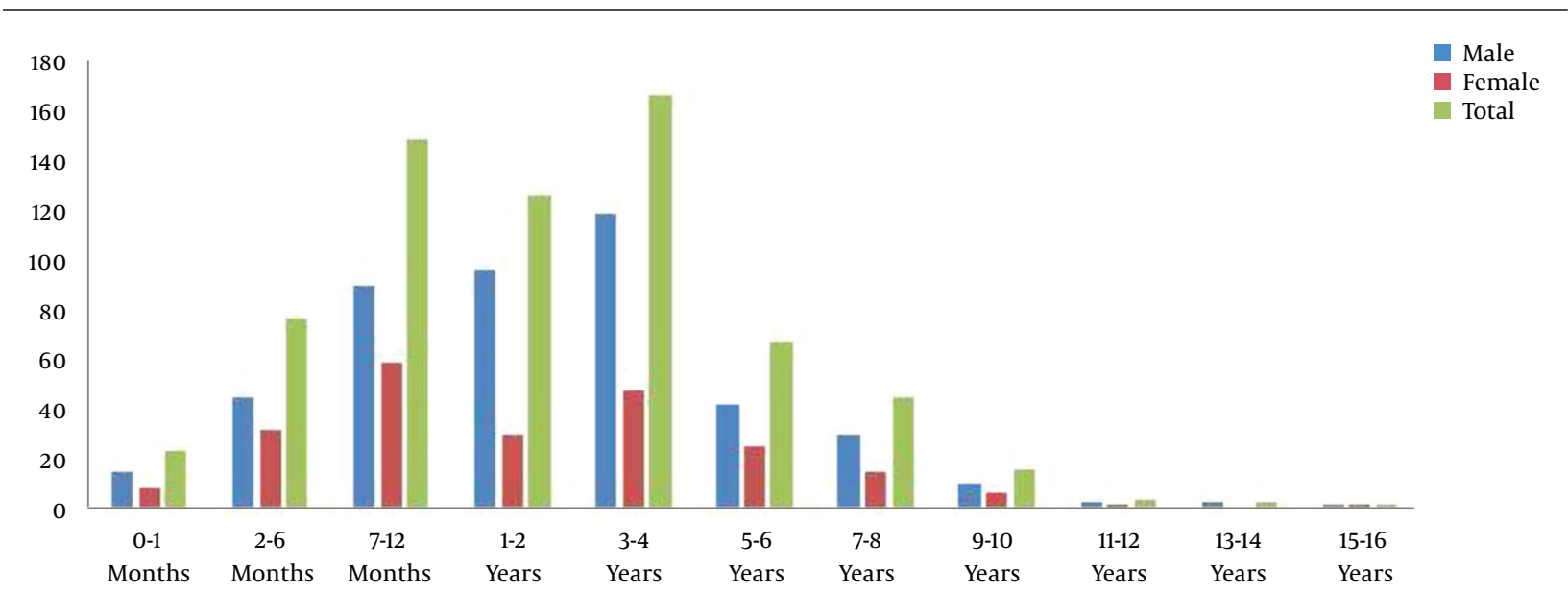

Figure 1. Age and sex distribution among patients who underwent FFB

two cases. Transient but significant oxygen desaturations (with an $\mathrm{O}_{2}$ nadir of $40 \%$ ) were seen in $21(2.62 \%$ ) patients. In most patients, increases in oxygen supplementation improved the desaturations. Severe desaturations occurred in five patients, in which two of them were ventilated during the FFB procedure. There was a significant correlation between patients with a history of exposure to cigarette smoking and oxygen desaturation during bronchoscopy $(\mathrm{P}=0.001)$. In 12 patients, the bronchoscope was required to be removed to improve oxygenation. Two patients experienced laryngospasm during the FFB procedure and three patients developed bronchospasm postoperatively. In four patients who developed minor hemorrhage, suctioning was successfully performed. Lastly, one child experienced an episode of apnea in the recovery room requiring mask ventilation.

\section{Discussion}

The present study aimed to investigate common indications, diagnostic yield and complications of FFBs performed at a tertiary care University hospital in Tehran, Iran, over a course of 5 years. To our knowledge, this is a first report describing indications and highlights possible complications of FFB procedure among pediatric population in our country. The use of FFB has revolutionized the investigation of pediatric airway problems and has resulted in an increased diagnostic yield. These indications have been developed following the need for assessment of clinical and radiological anomalies that cannot be otherwise evaluated by non-invasive methods. While FFB is currently considered as an accepted diagnostic tool for the pediatric population, it carries a number of adverse events dur-

\begin{tabular}{|c|c|c|}
\hline Indication & No. $(\%)$ & $\begin{array}{c}\text { Abnormal } \\
\text { Findings (\%) }\end{array}$ \\
\hline \multicolumn{3}{|l|}{ Chronic cough } \\
\hline Hemoptysis & $35(4.3)$ & $21(3.8)$ \\
\hline \multicolumn{3}{|l|}{ Airway obstruction } \\
\hline Stridor & $126(18.3)$ & $78(14.2)$ \\
\hline Persistent wheezing & $76(9.5)$ & $67(12.2)$ \\
\hline \multicolumn{3}{|l|}{ Radiographic abnormalities } \\
\hline $\begin{array}{l}\text { Persistent/ recurrent pneumonia or } \\
\text { consolidation }\end{array}$ & $124(15.5)$ & $92(16.8)$ \\
\hline Persistent atelectasis & $83(10.3)$ & $48(8.7)$ \\
\hline Localized pulmonary hyperlucency & $33(4.1)$ & $22(4.02)$ \\
\hline \multicolumn{3}{|l|}{ Therapeutic applications } \\
\hline Extraction of foreign bodies & $228(28.5)$ & $156(28.5)$ \\
\hline Administration of drugs & $4(0.5)$ & $1(0.18)$ \\
\hline $\begin{array}{l}\text { Removal of airway secretions or } \\
\text { mucus plugs }\end{array}$ & $11(1.3)$ & $5(0.91)$ \\
\hline Difficult intubations & $4(0.5)$ & $1(0.18)$ \\
\hline Cystic fibrosis & $32(4)$ & $27(4.93)$ \\
\hline Obtaining bronchoalveolar lavage & $44(5.5)$ & $29(5.3)$ \\
\hline Total & $800(100)$ & $547(100)$ \\
\hline
\end{tabular}

ing or after the procedure performed. In a recent survey being conducted by the Paediatric Bronchology Group of the European Respiratory Society (ERS) (13), it was shown that the overall complication rate ranged from $10 \%$ to $50 \%$, with the highest rate being observed in patients with postbronchoscopy related side effects such as bleeding, bronchospasm, laryngospasm, and reactions to medication. In 


\begin{tabular}{lcc}
\hline \multicolumn{1}{l}{ Table 2. Complications During and After Bronchoscopy } & \\
\hline Complication & No. of Patients & \% of FFBs \\
\hline Major & & \\
\hline \multicolumn{1}{|l}{ Pneumothorax } & 2 & 0.25 \\
\hline Massive bleeding $(>50 \mathrm{~mL})$ & 4 & 0.5 \\
\hline Respiratory failure & 1 & 0.12 \\
\hline Total & 7 & 0.87 \\
\hline Minor & & \\
\hline Hypoxia & 21 & 2.62 \\
\hline Bronchospasm & 3 & 0.37 \\
\hline Laryngospasm & 2 & 0.25 \\
\hline Total & 26 & 3.25 \\
\hline
\end{tabular}

the present study, the reported rates of complications and mortality were more favorable than those reported in the literature indicating the safety of FFB and its routine procedures $(14,15)$.

Aspiration of foreign bodies is a frequent pediatric emergency in which the adequacy of ventilation is the primary concern $(16,17)$. The rate of foreign body removal comprised about $30 \%$ of all FFBs performed in this study which was comparable to previous studies (16-18). A detailed history and physical examination is required for diagnosis of foreign body aspiration. In cases with negative imaging finding, a prompt bronchoscopy should be carried out. It should be noted that mother's information is critical, as they commonly report that a foreign object had been swallowed by their child. Patient's age and physical position at the time of ingestion is also an important clinical clue and is occasionally associated with the location of foreign body. The frequency of foreign body in either main bronchus is equal until the age of 15 years due to similar angles formed by the trachea and main bronchi by that age. The best treatment option is prevention, indicating the importance of public awareness and education regarding the risk of foreign body aspiration and standard ageappropriate toys and foods.

In the present study, FFB related complications were uncommon. The reported rates of minor and major complications were $0.25 \%$ and $0.87 \%$, respectively. No cases of fatality were reported. Also, there was no patient who failed to complete the FB procedure due to the occurrence of complications in our studied population. Overall, the rate of severe complications was found to be low $(n=6$, $0.03 \%$ ), and mostly occurred in children aged less than 5 years. Severe complication such as hypoxemia only occurred in two patients, whereas milder adverse events including stridor, fever and mild desaturation were found in $14 \%$ of our patients. A good understanding of procedural complications is essential as it can guide clinicians for appropriate management and improve preparedness in counseling parents.

In our study, non-life-threatening complications were developed in $24 \%$ of patients, in which transient desaturation comprised the most common abnormality observed in patients admitted to hospital. Definition for desaturation has been widely variable in prior studies. In a study done by Nussbaum (19), desaturation was defined as a drop in $\mathrm{SpO}_{2}$ to $65 \%-80 \%$, while in another study (7), a reduction in $\mathrm{SpO}_{2}$ up to $10 \%$ or more was considered as desaturation. Desaturation is a potentially dangerous complication as it can rapidly progress to bradycardia and finally lead to cardiac arrest. Prompt response is therefore required in order to avoid this complication.

In the present study, the diagnostic yield forchronic cough was low. This finding was similar to previous studies $(13,20)$, suggesting that a normal bronchoscopy can be of great reassurance for parents whom their child had a persistent cough regardless of medical management.

Our study has a number of limitations. Failure to enroll all cases undergoing bronchoscopy during the study period may have had the potential to introduce bias. In particular, an informed consent was less likely to be obtained from certain patient populations including younger individuals and emergency conditions. Another possible limitation of the present study was the potential for biased selection, which is related to the retrospective nature of the study. Therefore, further prospective studies in the future may be needed to highlight the new diagnostic bronchoscopy interventions. In conclusion, FFB is a valuable diagnostic tool in the management of airway anomalies among pediatric population. Our study showed that the procedure is associated with an acceptable safety profile with low short and long term complications. Furthermore, the capacity to obtain BAL samples with this procedure increases its diagnostic yield which in turn can play an integral role in identifying the exact pathology and best treatment option. Our findings support early intervention and utilization of bronchoscopy in the pediatric population with variable respiratory complaints.

\section{Footnotes}

Authors' Contribution: Critical revision of the manuscript, Study concept and design: Rohola Shirzadi and Mohammad Reza Modaresi; acquisition, analysis and interpretation of data, drafting of the manuscript: Niloofar Razavi Khorasani; participation in the data acquisition: Safoura Navaei, Farzad Masiha, Seyed Hossein Mirlohi, 
and Mahya Mohamadi; analysis, interpretation of data, statistical analysis: Alireza Ebrahimsoltani.

Conflict of Interests: The authors have no conflict of interest to declare.

Ethical Approval: The study was approved by the Ethics Committee of Tehran University of Medical Sciences. The process of this study was performed in accordance with the Declaration of Helsinki and other applicable guidelines, laws, and regulations.

Funding/Support: There is no financial support statement.

Patient Consent: All parents were asked to fill an informed consent before the procedures were performed.

\section{References}

1. Navarro Rojas AA. Bronchoscopy in children in South America. Paediatr Respir Rev. 2006;7(4):288-92. doi: 10.1016/j.prrv.2006.04.011. [PubMed: 17098644]

2. Nicolai T. Pediatric bronchoscopy. Pediatr Pulmonol. 2001;31(2):15064. doi: 10.1002/1099-0496(200102)31:2<150::aid-ppul1024>3.0.co;2-6. [PubMed: 11180692].

3. Wood RE, Fink RJ. Applications of flexible fiberoptic bronchoscopes in infants and children. Chest. 1978;73(5 Suppl):737-40. doi: 10.1378/chest.73.5_supplement.737. [PubMed: 639589].

4. Webster I, Goussard P, Gie R, Janson J, Rossouw G. The indications and role of paediatric bronchoscopy in a developing country, with high prevalence of pulmonary tuberculosis and HIV. Expert Rev Respir Med. 2017;11(2):159-65. doi: 10.1080/17476348.2017.1280397. [PubMed: 28107788].

5. Vega-Briceno LE, Holmgren NL, Bertrand P, Rodriguez JI, Barriga F, Contreras I, et al. [Utility of bronchoalveolar lavage in immunocompromised children: Diagnostic yield and complications]. Arch Bronconeumol. 2004;40(12):570-4. Spanish. doi: 10.1016/s15792129(06)60377-7. [PubMed: 15574271].

6. Rosenthal M. Bronchoscopy and infection. Paediatr Respir Rev. 2003;4(2):143-6. doi: 10.1016/s1526-0542(03)00025-3.

7. Rodriguez Martinez C, Sossa MP. [Factors associated with complications caused by bronchoscopy in pediatric patients]. Arch Bronconeumol. 2003;39(11):501-6. Spanish. doi: 10.1016/s0300-2896(03)75440-4. [PubMed: 14588203].

8. Schnapf BM. Oxygen desaturation during fiberoptic bronchoscopy in pediatric patients. Chest. 1991;99(3):591-4. doi: 10.1378/chest.99.3.591. [PubMed: 1995213].
9. Schellhase DE, Tamez JR, Menendez AA, Morris MG, Fowler GW, Lensing SY. High fever after flexible bronchoscopy and bronchoalveolar lavage in noncritically ill immunocompetent children. Pediatr Pulmonol. 1999;28(2):139-44. doi: 10.1002/(sici)10990496(199908)28:2<139::aid-ppul10>3.0.co;2-2. [PubMed: 10423314].

10. Pue CA, Pacht ER. Complications of fiberoptic bronchoscopy at a university hospital. Chest. 1995;107(2):430-2. doi: 10.1378/chest.107.2.430. [PubMed: 7842773].

11. Manna SS, Durward A, Moganasundram S, Tibby SM, Murdoch IA. Retrospective evaluation of a paediatric intensivist-led flexible bronchoscopy service. Intensive Care Med. 2006;32(12):2026-33. doi: 10.1007/s00134-006-0351-y. [PubMed: 16941167].

12. de Blic J, Midulla F, Barbato A, Clement A, Dab I, Eber E, et al. Bronchoalveolar lavage in children. ERS Task Force on bronchoalveolar lavage in children. European Respiratory Society. Eur RespirJ. 2000;15(1):217-31. doi:10.1183/09031936.00.15121700. [PubMed: 10678650].

13. Barbato A, Magarotto M, Crivellaro M, Novello A Jr, Cracco A, de Blic J, et al. Use of the paediatric bronchoscope, flexible and rigid, in 51 European centres. Eur Respir J. 1997;10(8):1761-6. doi: 10.1183/09031936.97.10081761. [PubMed: 9272916].

14. Terkawi RS, Altirkawi KA, Terkawi AS, Mukhtar G, Al-Shamrani A. Flexible bronchoscopy in children: Utility and complications. Int J Pediatr Adolesc Med.2016;3(1):18-27. doi:10.1016/j.ijpam.2015.12.003. [PubMed: 30805463]. [PubMed Central: PMC6372410].

15. Tang LF, Chen ZM. Fiberoptic bronchoscopy in neonatal and pediatric intensive care units: A 5-year experience. Med Princ Pract. 2009;18(4):305-9. doi: 10.1159/000215729. [PubMed: 19494539].

16. Haddadi S, Marzban S, Nemati S, Ranjbar Kiakelayeh S, Parvizi A, Heidarzadeh A. Tracheobronchial foreign-bodies in children; a 7 year retrospective study. Iran J Otorhinolaryngol. 2015;27(82):377-85. [PubMed: 26568942]. [PubMed Central: PMC4639691].

17. Rodriguez H, Passali GC, Gregori D, Chinski A, Tiscornia C, Botto $\mathrm{H}$, et al. Management of foreign bodies in the airway and oesophagus. Int J Pediatr Otorhinolaryngol. 2012;76 Suppl 1:S84-91. doi: 10.1016/j.ijporl.2012.02.010. [PubMed: 22365376].

18. Saquib Mallick M, Rauf Khan A, Al-Bassam A. Late presentation of tracheobronchial foreign body aspiration in children.J Trop Pediatr. 2005;51(3):145-8. doi: 10.1093/tropej/fmh103. [PubMed: 15831667].

19. Nussbaum E. Pediatric fiberoptic bronchoscopy: Clinical experience with 2,836 bronchoscopies. Pediatr Crit Care Med. 2002;3(2):171-6. doi: 10.1097/00130478-200204000-00015. [PubMed:12780989].

20. Kirvassilis F, Gidaris D, Ventouri M, Zampouri A, Mylona M, Keramidiotis A, et al. Flexible fiberoptic bronchoscopy in Greek children. Hippokratia. 2011;15(4):312-5. [PubMed: 24391411]. [PubMed Central: PMC3876845]. 